\title{
Use of the experimental modal analysis for analytical lifetime estimation of a bogie frame
}

\author{
Ion Manea $^{1 *}$, Ioan Sebesan ${ }^{2}$, Mihai Matache ${ }^{3}$, Gabriel Prenta ${ }^{1}$ and Catalin Firicel ${ }^{1}$ \\ ${ }^{1}$ SC Softronic SRL, Calibration and Mechanical Testing Laboratory, Craiova, No.40, Dolj, Romania \\ ${ }^{2}$ Polytechnic University of Bucharest, Sector 6, Splaiul Independentei, No. 313, Romania \\ ${ }^{3}$ INMA Bucharest, Sector 1, Blv. Ion Ionescu de la Brad, No. 6, Romania
}

\begin{abstract}
The fatigue strength validation by tests of the railway bogie frames requires existence of an expensive laboratory and a long time for testing of about 6 to 10 months. Considering these aspects, the European norms admit that fatigue tests can be replaced by finite elements analysis (FEA), with the condition that analytical model to be correctly realized and validated by tests. Experimental modal analysis (EMA) provides a powerful tool for validation of the FEA model by experimental data. The article presents an application for assessment of the fatigue strength of a three-axle locomotive bogie frame. Using Ansys, was carried out the structural analysis of the bogie frame, resulting the modal model characterized by the modal parameters: Eigen frequencies and Eigen shapes. The analytical model was validated by an EMA application carried out on the bogie frame and correlation analysis of the EMA and FEA models. Using a special measuring wheel set, were determined the wheelrail interaction forces for various locomotive running conditions. The analytical structural model, validated through experimental data, and the data files containing the wheel-rail interaction forces, constitutes the input data for the nCode program that evaluates the bogie frame lifetime using appropriate stress curves and a recognized accumulation of damage hypothesis, e.g. the Palmgren-Miner.
\end{abstract}

\section{Background of EMA and FEA analysis}

Experimental Modal Analysis (EMA) is the procedure of building the mathematical model of a structure based on the experimental data obtained by measurements made on the structure brought into a controlled vibrational state.

The system is excited under well-defined conditions and, determining the evolution laws of excitation and vibrational response is identified the modal model characterized by a minimum number of modal parameters, describing its vibration Eigen modes: Eigen frequencies, damping factors and Eigen shapes.

The most general EMA mathematical model which describes the dynamics of a system with $\mathrm{N}$ degrees of freedom (DOF) is formulated as a system of differential equations adapted to the calculation method, which can be in time or in frequency domain. Equation

${ }^{*}$ Corresponding author: ion.manea@softronic.ro 
(1) shows the general form of the system moving equation for the time domain, and equation (2) shows the general form of the moving equation for the frequency domain [1].

$$
\begin{gathered}
{[M]\{\ddot{x}(t)\}+[C] \cdot\{\dot{x}(t)\}+[K]\{x(t)\}=\{F(t)\}} \\
-\omega^{2}[M]\{X(\omega)\}+i \omega[C] \cdot\{X(\omega)\}+[K]\{X(\omega)\}=\{Q(\omega)\}
\end{gathered}
$$

The unknown matrices elements of mass, stiffness and damping must first be estimated from experimental data, through measurements carried out in time or frequency domains. Once the matrices elements were estimated, the modal parameters are found as a solution of an eigenvectors and eigenvalues problem.

Corresponding to equations(1) and (2), the most mathematical models, for description of dynamic response, can be arranged in a form of unit impulse response functions, $h_{p q}(t)$, or frequency response functions, $H_{p q}(\omega)$, in such a way to be described by the following relations in the time (3) or frequency domain (4) :

$$
\begin{gathered}
h_{p q}(t)=\sum_{r=1}^{N} A_{p q r} e^{\lambda_{r} t}+A_{p q r}^{*} e^{\lambda_{r}^{*} t} \\
H_{p q}(\omega)=\sum_{r=1}^{N} \frac{A_{p q r}}{i \omega-\lambda_{r}}+\frac{A_{p q r}^{*}}{i \omega-\lambda_{r}^{*}}
\end{gathered}
$$

Unit impulse response functions are rarely or never directly measured, but they are calculated from associated frequency response functions (FRF) via the inverse FFT.

It have been used the following notations:[M], [C], [K], the mass, damping and stiffness matrices; $\{\ddot{x}(t)\},\{\dot{x}(t)\},\{x(t)\}$, the vectors of acceleration, velocity and displacement; $\{F(t)\}$ generalized vector of forces; $\omega$, angular frequency $(\mathrm{rad} / \mathrm{sec}) ; \mathrm{p}$, measured DOF of response; $\mathrm{q}$, measured DOF of input; $\mathrm{r}$, modal number vector; N, number of modal frequencies; $A_{p q r}$, residue for mode $\mathrm{r} ; \lambda_{r}$, system pole for mode $\mathrm{r}$.

In terms of Finite Element Analysis (FEA), the structure is divided into finite elements between which the mathematical connections are established, equivalent to the physical connections between the adjacent elements or the environment. To the physical model corresponds a mathematical model, similar to the mathematical model of EMA, the difference being that in the FEA model are not considered the damping of system?

$$
[M] \cdot\{\ddot{x}\}+[K] \cdot\{x\}=\{0\}
$$

Solving the system of differential equations leads to the analytical determination of the Eigen frequencies and Eigen shapes which, referring to the same system, must to be the same as those determined by experimental EMA.

Finite element analysis works with an approximate mathematical model, realized by more or less gross approximations of both geometric and material characteristics.

Even if the mathematical model is perfect, it will still provide an approximate analytical model, because it is known that the characteristics of the material used in the modelling never coincide with the real characteristics of the structure.

In addition, it is well known that any geometrical modelling of a product is accompanied by more or less gross execution errors, errors that cannot be translated into geometric model.

It follows, from the above, that a good and reliable analytical model can only be obtained by validation with the data provided by the real system through the EMA. Only an analytical model validated through experimental data can be used with confidence. 


\section{FEA and EMA analysis of a three-axle locomotive bogie frame}

Finite Element Analysis. It was been used the SolidWorks for geometrical modelling and Modal and Structural modules of Ansys 15 for modal and structural analysis of bogie frame.

Analysis was made in linear elastic regime, the bogie frame being modelled as follows:

- Tetrahedral elements type Solid 92 in the number of: 806471 elements and 1490919 nodes.

- Linear rigid beam elements type Beam, for the structure between the primary suspension and axle, for axle, and for the forces transmission from the application points to the structure.

- Spring elements for the primary and secondary suspensions.

The analytical modal analysis was performed in the frequency range of 10 to $100 \mathrm{~Hz}$. Modal shapes, are presented in the correlation analysis section in connection with the EMA.

Experimental Modal Analysis. EMA tests were carried out inSoftronic CraiovaRomania on a three-axle bogie frame designed for LEMA $6000 \mathrm{~kW}$ electric locomotive. The bogie frame has been redesigned for extended range of operating temperatures of $-40^{\circ} \mathrm{C}$ to $+40^{\circ} \mathrm{C}$. The main material used is steelS355NL. To perform the EMAtests, the bogie frame was placed on four helicoidally springs, each having elastic constant $k=519 \mathrm{~N} / \mathrm{mm}$, in normal operating position, the bogie frame can be considered as a free system, without restricting anyDOF.

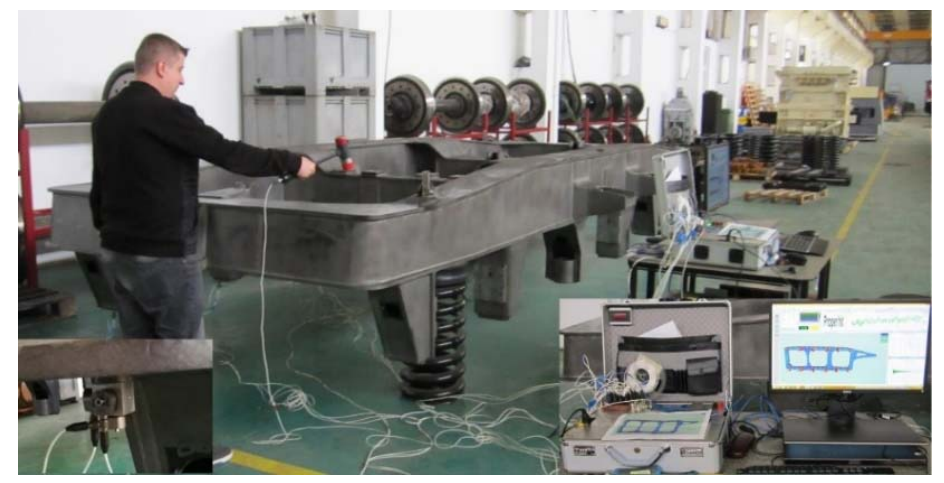

Fig. 1. Mounting of the three-axle locomotive bogie frame for EMA tests.

The excitation of the bogie frame was made in only one point using the impact method with an impact hammer of $25 \mathrm{kN}$. It was used a mediation over 4impacts to eliminate the noise in measurements and to obtain a high accuracy of FRF. The analysis was made in frequency range of 10 to $100 \mathrm{~Hz}$. The acceleration response was measured in a number of 45 points, for each measuring point the response being measured on three orthogonal directions: vertical, transverse and longitudinal, resulting a number of $135 \mathrm{DOF}$ measured. The points of measurement have been selected to allow a suggestive animation of the structure.

For EMA tests was used the following excitation and measuring equipment and software:

-Piezoelectric accelerometers type 355B03, $10.19 \mathrm{mV} /\left(\mathrm{m} / \mathrm{s}^{2}\right), \pm 490 \mathrm{~m} / \mathrm{s}^{2}, 15 \mathrm{pcs}$;

-Impact Hammer type 086D20, $0.23 \mathrm{mV} / \mathrm{N}, \pm 22240 \mathrm{~N}$ pk, 1 pcs.;

-Softronic Data Acquisition System including: LAN-XI type 3053B120, 12 input chann., $25.6 \mathrm{kHz}$ and LAN-XI type 3160A042, 4 input chan., 2output chan.,25.6 kHz;

-Structural Dynamics Test Consultants, a software working under Pulse Lab shop to provide a dedicated measurement environment for structural dynamic measurements; 
-Pulse Reflex Modal Analysis, a post-processing module for classical modal analysis, using hammer or shaker excitation. It works with data measured as FRF and express the Eigen modes by modal parameters: damped frequency, damping, and residues;

-Pulse Reflex Correlation Analysis, provides a powerful tool to verify and validate the FEA model by true EMA modal model. The correlation analysis is accomplished by graphical comparisons, shape animations and numerical Modal Assurance Criterion (MAC).

Both modal models, FEA and EMA, were identified in the frequency range of 10 to $100 \mathrm{~Hz}$ and the correlation analysis of the two models was performed. Figure 2 presents the vector comparison panel with Cross Modal Assurance Criterion for the two models. Table 1 presents the results of FEA and EMA correlation analysis. There are presented the damped frequencies for the two modal models and the evaluation error of analytical damped frequency. It is presented the MAC criterion of modal shapes for the two models. Figures 3 to 7 presents the frames in the first five Eigen modes. Analyzing the presented data it can be concluded that:

-The damped frequency error estimated by FEA is less than 5\%compared to EMA;

-The MAC correlation criterion is greater than 0.6;

-Modal shapes calculated by FEA are very similar to modal shapes calculated by EMA.

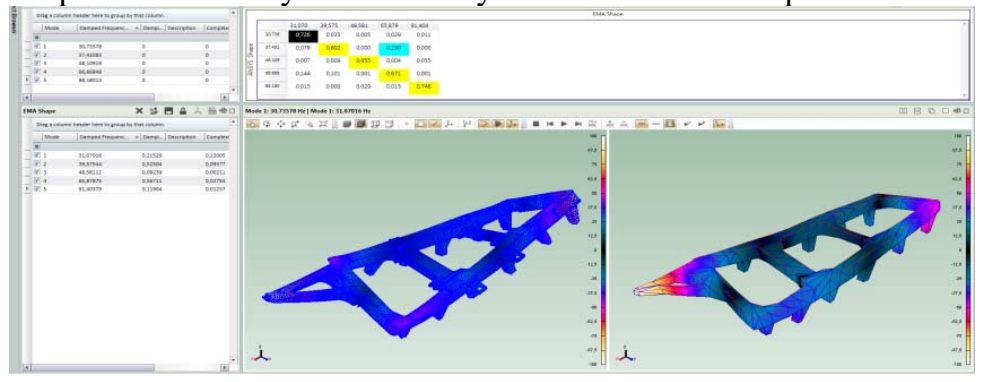

Fig. 2. Pulse Reflex Correlation- panel for Modal Assurance Criterion CrossMAC comparison.

Table 1. Results of FEA - EMA correlation analysis.

\begin{tabular}{|c|c|c|c|c|c|c|c|}
\hline \multirow{2}{*}{$\begin{array}{l}\text { Mod } \\
\text { No. }\end{array}$} & FEA & \multicolumn{3}{|c|}{ EMA } & \multirow{3}{*}{$\begin{array}{c}\text { Mode } \\
\text { direction }\end{array}$} & \multirow{2}{*}{$\begin{array}{c}\text { Frequency } \\
\text { Error }\end{array}$} & \multirow{3}{*}{$\begin{array}{c}\text { MAC } \\
\text { Modal } \\
\text { Assurance } \\
\text { Criterion }\end{array}$} \\
\hline & $\begin{array}{l}\text { Damped } \\
\text { Frequency }\end{array}$ & $\begin{array}{l}\text { Damped } \\
\text { Frequency }\end{array}$ & Damping & Complexity & & & \\
\hline & $(\mathrm{Hz})$ & $(\mathrm{Hz})$ & $(\%)$ & & & $(\%)$ & \\
\hline 1 & 30.73 & 31.07 & 0.215 & 0.120 & Vertical & -1.106 & 0.726 \\
\hline 2 & 37.43 & 39.57 & 0.525 & 0.098 & Vertical & -5.717 & 0.602 \\
\hline 3 & 48.11 & 48.58 & 0.092 & 0.002 & Transverse & -0.998 & 0.655 \\
\hline 4 & 66.66 & 65.87 & 0.567 & 0.027 & Vertical & 1.185 & 0.671 \\
\hline 5 & 88.18 & 91.40 & 0.119 & 0.012 & Transverse & -3.652 & 0.746 \\
\hline
\end{tabular}
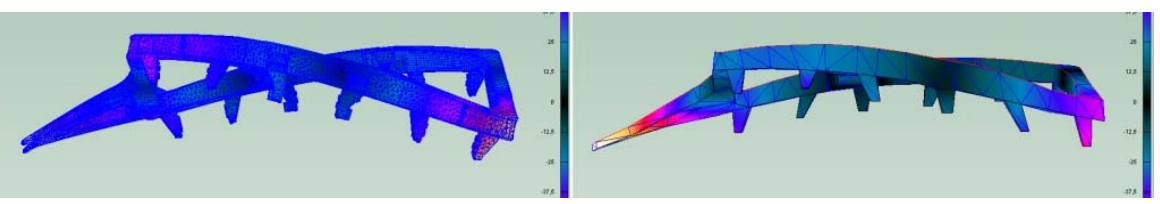

Fig. 3. The $1^{\text {st }}$ eigenmodes, OZ direction, left FEA $30.73 \mathrm{~Hz}$, right EMA $31.07 \mathrm{~Hz}$.
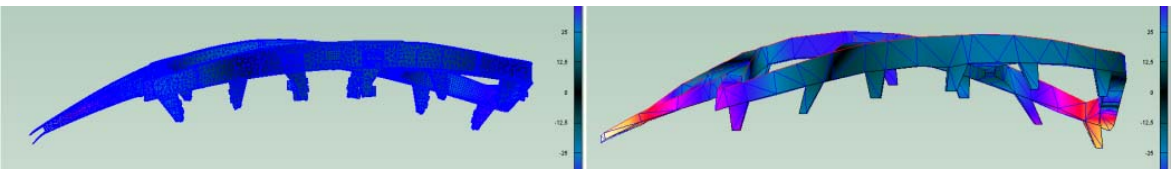

Fig. 4. The 2 $2^{\text {nd }}$ eigenmodes, $\mathrm{OZ}$ direction, left FEA $37.43 \mathrm{~Hz}$, right EMA $39.57 \mathrm{~Hz}$. 

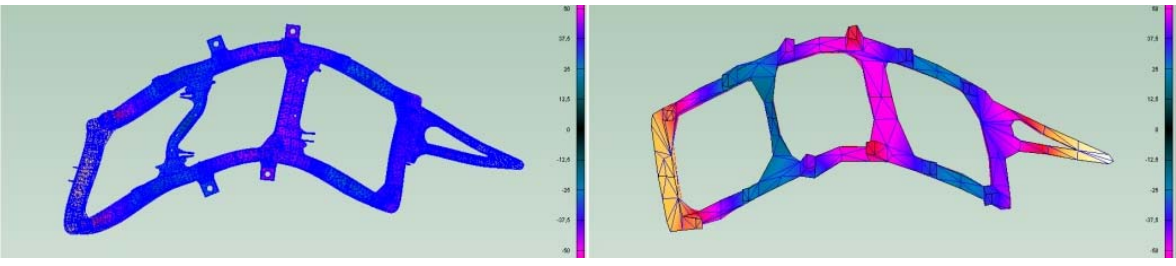

Fig. 5. The $3^{\text {rd }}$ eigenmodes, OY direction, left FEA $48.11 \mathrm{~Hz}$, right EMA $48.58 \mathrm{~Hz}$.

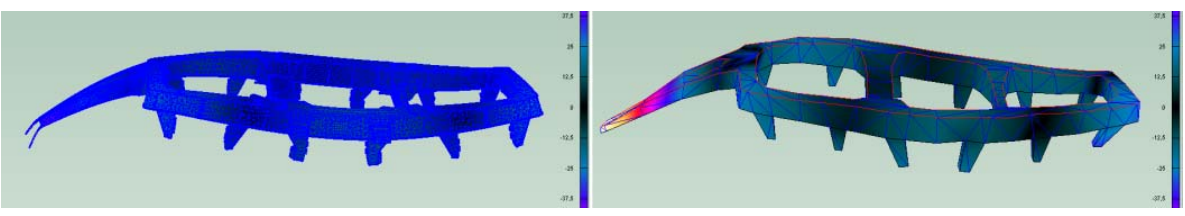

Fig. 6. The $4^{\text {th }}$ eigenmodes, OZ direction, left FEA $66.66 \mathrm{~Hz}$, right EMA $65.87 \mathrm{~Hz}$.
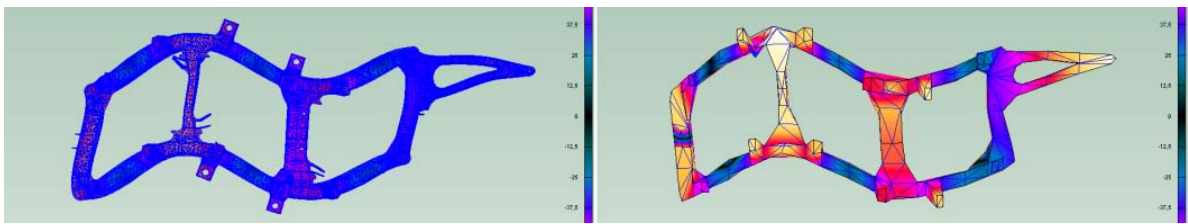

Fig. 7. The $5^{\text {th }}$ eigenmodes, Oy direction, left FEA $88.18 \mathrm{~Hz}$, right EMA $91.40 \mathrm{~Hz}$.

Considering the data in Table 1 and modal shapes shown in Figures $3 \div 7$, it can be concluded that the FEA analytical model is a correctly calibrated model and can be used to evaluate the dynamic response of the bogie frame.

\section{Lifetime estimation of the bogie at normal operating conditions}

The fatigue of a structure is caused by loading the structure with repetitive stresses for a time.

The traditional method to estimate the number of cycles of a repetitive stresses that a structure can withstand, use the empirically data from a large number of tests on various types of material subject to repetitive stress cycles. This technique states that the amplitude of the stresses to which a material is subjected is linked by an empirical curve to the number of expected repetitive stress cycles until the fatigue failure. For many types of material are determined such empirical curves, known as the S-N or Wholer curves. This technique is still used today and is known as the „stress-life” approach. The lifetime estimation by stress-life approach uses the hypothesis that the stress remains in the elastic region, therefore the stress-life approach is suitable in fatigue analysis for large number of stress cycles, $\mathrm{N} \geq 10^{3}$.

If it is defined a load time history with variable amplitude of stresses, several steps must be taken to calculate the lifetime from stresses applied to the individual cycles. In a first stage, the history of the load is decomposed into peak-valley sequences. Next, the reduced load sequence is processed with a standard cycle counting algorithm (like rain flow counting algorithm) and extracts individual stress cycles. Single stress cycle are then used together with the S-N curves to calculate the lifetime associated with each stress cycle.

After a lifetime (or damage) is calculated for each individual stress cycle, it is necessary a hypothesis for determining the total lifetime, or cumulative damage. The most commonly hypothesis of damage assessment is based on the cumulative damage rule, or the Palmgren- 
Miner rule, which states that the total damage accumulated in the stress history is given by the linear sum of the partial damage generated by the individual cycle loads:

$$
D_{\text {total }}=\sum_{i=1}^{N} D_{i} \quad \text { or } \quad \frac{1}{N_{\text {total }}}=\sum_{i=1}^{N} \frac{1}{N_{i}}
$$

Where $\mathrm{N}$ is the lifetime, expressed in repeated stress cycles, and $\mathrm{D}$ is the damage.

For the bogie frame, the main external loads come from the railway and are constituted by the wheel-rail interaction forces: transverse forces, Y, and vertical forces, Q. In order to determine these loads, within Softronic was developed a measuring wheel set for measurement of wheel-rail interaction forces. With the LEMA $6000 \mathrm{~kW}$ locomotive equipped with the measuring wheel set were carried out several on-track tests to determine the time history of wheel-rail interaction forces, results being saved in the data files $[2,3]$.

With the FEA model, validated by EMA experimental data, was run a static analysis, the bogie frame being subject to action of average operating Y/Q loads, experimental determined. The results of stress distribution on the bogie frame were saved as file with .rst extension.

Data files containing interaction forces and .rst file, constitutes inputs data for the HBM Prenscian Codes software, module Design Life, which evaluates fatigue damage and lifetime on the basis of Wholer curves and Palmgren-Miner hypothesis of cumulative damage.

Figure 8 presents the logical scheme to evaluate the service lifetime of bogie frame. From records of $\mathrm{Y} / \mathrm{Q}$ interaction force, it was selected a record, in alignment, at the speed of $80 \mathrm{~km} / \mathrm{h}$, with a length of $1 \mathrm{~km}$. In Figure 8 , upper left, is presented the time history of forces Y/Q. The upper center presents the results of running of Rain flow module that gives the statistical distribution of stress cycles related to average value and the stress level. In the upper right, are shown the stress distribution on the bogie frame, for the original static load and for the dynamic loading with Y/Q forces. The lower table shows the numerical results of analysis, with distribution of main parameters on nodes or elements. The lifetime of each node/element is presented. It can be seen from the table that for the most stressed areas of the frame, the lifetime is of approx. $4.79 \times 10^{9}$ lengths of record, or equivalent approx. $4.79 \times 10^{9} \mathrm{~km}$ in alignment running with a speed of $80 \mathrm{~km} / \mathrm{h}$.

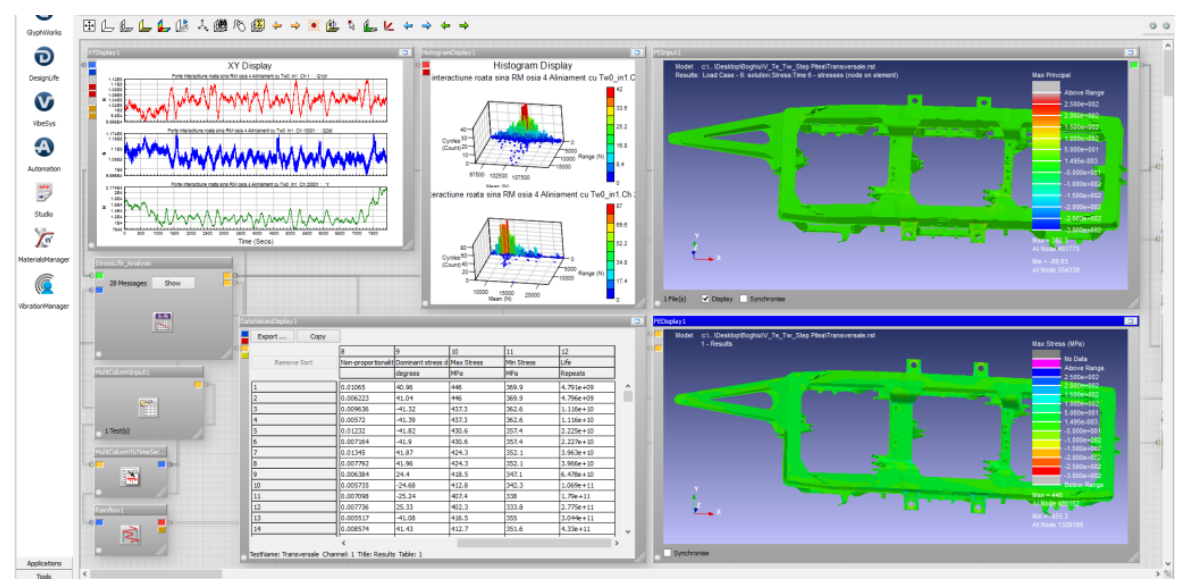

Fig. 8. Logical scheme to evaluate the service lifetime of the LEMA $6000 \mathrm{~kW}$ bogie frame. 


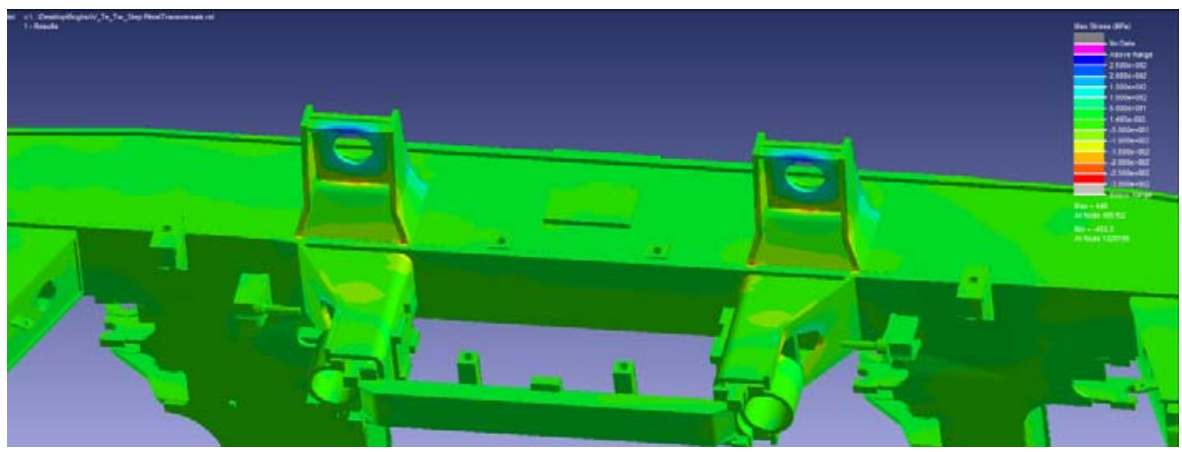

Fig. 9. Detail for stress distribution, in $\mathrm{MPa}$, in the most stressed areas of the bogie frame.

\section{Conclusions}

The article presents a method for validating the FEA analytical model of a bogie frame through the real modal model realized by experimental modal analysis, EMA. The validated analytical model is used to assess the lifetime of the bogie frame, corresponding to the on-track running of bogie, under experimentally determined running conditions.

At the moment, the method outlined in the paper is under analysis and development.

This work was supported by a grant of the Romanian National Authority for Scientific Research and Innovation, CNCS-UEFISCDI, project number PN-III-P2-2.1-PTE-2016-0008, within PNCDI III

\section{References}

1. D.J. Ewins, Modal Testing, Theory, Practice, John Wiley and Sons, Inc., New York 1984

2. I. Manea, I Sebesan and all, System for measurement of interaction forces between wheel and rail for railway vehicles, Matec Web of Conferences 137, 01006 (2017)

3. EN 13749, Railway applications - Wheelsets and bogies - Method of specifying the structural requirements of bogie frames, March 2011 